\title{
Frederik Barkhof, Nick C. Fox, Antonio J. Bastos-Leite and Philip Scheltens: Neuroimaging in dementia. 1st edition
}

\author{
Springer, e-version, around $£ 110 ; 297$ pages
}

\author{
Ravi V. Jampana
}

Received: 3 October 2011 / Accepted: 6 January 2012 / Published online: 31 January 2012

(C) Springer-Verlag 2012

This is an excellent, comprehensive first edition book on practical use of neuroimaging in dementia. There is particular emphasis on MRI and its advanced techniques, but this book has incorporated all the relevant functional imaging techniques (SPECT and PET) as well. In keeping with the advances in the understanding of dementia, its classification and imaging techniques, the now nearly 10 -year-old textbook Magnetic Resonance in Dementia has been completely rewritten, expanded with addition of new techniques, images and aptly re-titled.

Everyday language with use of diagrams, flow charts and coloured text boxes highlighting "take home" information gives the feeling of listening to a good lecture rather than just reading a book. Clinical back ground, appropriate histopathology, clinical criteria and classification of dementia

R. V. Jampana $(\bowtie)$

Department of Neuroradiology, Institute of Neurological Sciences, Southern General Hospital,

1345 Govan Road,

Glasgow G51 4TF, UK

e-mail: Ravi.Jampana@ggc.scot.nhs.uk have been thoroughly covered which would help any radiologist with an interest in dementia but does not wish to dwell into the thicker and bigger textbooks on this hot topic. Information and protocols of various imaging techniques were elucidated in the next chapter. The readers are then taken through the all important chapter on "Normal ageing" before various conditions are divided conveniently into detailed chapters as primary grey matter loss, primary white matter loss and dementia with associated brain swelling covering all the important differentials. This approach is very practical for radiologists, and practical suggestions such as red flags on MRI, when to repeat imaging and a new diagnostic paradigm in dementia where imaging plays a crucial role also stood out in this book.

There is limited but good suggested reading at the end of each chapter subdivided under various conditions. Whilst the book may feel "text heavy" rather than images, it has enough examples of all the conditions. I thoroughly recommend this book to all radiologists, senior radiology trainees and clinicians with an interest in dementia. With the current interest in dementia and the increasing use of imaging, this would be essential reading to all radiologists. 